\title{
EVALUAR "PARA" EL APRENDIZAJE, APRENDER PARA ESTAR MOTIVADO: EL ORDEN DE LOS FACTORES SÍ AFECTA AL PRODUCTO
}

\author{
ASSESSMENT FOR LEARNING, TO LEARN TO BE MOTIVATED: \\ THE ORDER OF FACTORS ALTERS THE PRODUCT
}

\author{
Jesús Alonso Tapia* e Iván de la Red Fadrique**
}

Universidad Autónoma de Madrid

\section{RESUMEN}

Este estudio analiza los efectos de la introducción de nuevos procedimientos de evaluación en la motivación. 113 alumnos de Geografía ( $3^{\circ} \mathrm{ESO}$ ) recibieron enseñanza en la solución de problemas de Geografía mediante tareas que permitían identificar la raíz de sus dificultades y darles retroalimentación precisa. Posteriormente, en lugar de evaluar el conocimiento de conceptos y hechos, se evaluó la comprensión mediante tareas análogas a las utilizadas en el entrenamiento - tareas de transferencia-, y se examinó el valor motivacional que los alumnos atribuían al nuevo modo de evaluación. Los resultados muestran las ventajas y limitaciones del mismo y permiten extraer consecuencias para la Orientación Educativa.

Palabras clave: Motivación para aprender, entorno de aprendizaje, evaluación para el aprendizaje, orientación del profesorado,

\section{ABSTRACT}

This study analyses the effects of introducing new assessment procedures on learning motivation. 113 Secondary School students $\left(9^{\text {th }}\right.$ grade) were trained in applying geographical knowledge to solving Geography problems. Tasks used allowed to identify the causes of learning difficulties and to give precise feedback and help. Then, comprehension was assessed using new analogue tasks - transfer tasks- . Students were asked about interest and motivation aroused by the new assessment procedures. Results have shown both the positive effects, and limitations of these procedures, and have important implications for Educational Guidance.

\footnotetext{
* Jesús Alonso Tapia, es Profesor Titular de Evaluación Psicológica en la Universidad Autónoma de Madrid. Su línea de investigación se centra en la motivación, evaluación y autorregulación del aprendizaje. Puede accederse a muchas de sus publicaciones en http://www.uam.es/gruposinv/meva/ y en http://innova.decp.uam.es/main. php?id=198.

** Iván de la Red Fadrique.
} 
Key words: Learning motivation, Learning environment, learning assessment, teacher orientation.

\section{Problema y contexto teórico}

Un hecho que preocupa de modo reiterado a profesores, padres y educadores en general y en relación con el que se pide ayuda a menudo a los Servicios de Orientación es la falta de interés y esfuerzo de los alumnos por aprender o, lo que es igual, la falta de motivación. Este hecho plantea la pregunta "¿Qué puede hacerse para que los alumnos se esfuercen para comprender y aprender lo que les enseñamos y no sólo para aprobar?"

Responder a la cuestión anterior implica examinar los entornos de aprendizaje creados por los profesores y valorar el impacto motivacional de los mismos. Para ello es preciso tener en cuenta que tales entornos interactúan con las características con que los alumnos afrontan las situaciones de aprendizaje, ya que éstas parecen modular el impacto motivacional de aquellos.

Uno de los componentes del entorno de aprendizaje que más influye en la motivación y al que, sin embargo, menos atención se le presta a la hora de buscar soluciones es la evaluación de los aprendizajes. Por este motivo, en el presente artículo nos centraremos en el análisis del efecto de la evaluación sobre la motivación y en la búsqueda de vías de actuación sobre los problemas que plantea. Antes, sin embargo, puesto que las características con que los alumnos afrontan la actividad académica modulan el impacto de las distintas formas de actuación de los profesores - y la evaluación es una de ellas- (Alonso Tapia y Pardo, 2006), vamos a resumir de qué depende a nivel personal el interés y esfuerzo de los alumnos.

\section{Pilares de la motivación para el aprendizaje}

Varios trabajos y revisiones recientes confluyen básicamente en la descripción de los factores en que se apoya la motivación por aprender (Alonso Tapia, 2005a. Alonso Tapia y Ruiz, 2007, Eccles y Wigfield, 2002). De acuerdo con estos trabajos, el esfuerzo que un estudiante pone en la realización de tareas y actividades que pueden permitirle aprender depende, por un lado, de que tenga claro qué es lo que se pretende que aprenda con la realización de la tarea. Si no sabe qué debe aprender al realizarla, difícilmente va a esforzarse por aprender. Por otra parte, supuesto que el objetivo esté claro, la motivación se apoya en tres factores:

a) Las metas que persigue y que definen los beneficios que potencialmente le puede proporcionar el hecho de aprender lo que se le propone, metas que hacen que los alumnos afronten el trabajo con una motivación predominantemente intrínseca o extrínseca;

b) los efectos negativos que puede suponer el realizar el esfuerzo necesario para intentar aprender así como las consecuencias que pueden seguirse de no lograrlo, efectos que definen los costos del aprendizaje; y

c) las expectativas de conseguir los objetivos, ligadas al grado en que se cree que se sabe cómo lograrlos y en que se considera que este logro depende de uno mismo, creencias que definen las expectativas de autoeficacia y de control personal y que 
dependen, a su vez, de las experiencias de progreso del alumno ligadas en buena medida al grado en que "sabe pensar" y "autorregular" su proceso de aprendizaje.

Estos tres factores están relacionados formando un "trípode" en el que se apoya la motivación, de modo que cuando uno falla, el alumno deja de esforzarse. Finalmente, estos tres factores no actúan en el vacío, sino en el entorno que configura la actuación de profesores y padres.

Cuando tenemos que esforzarnos al hacer una tarea, no todas las metas hacen que lo hagamos en el mismo grado. Si lo que preocupa es evitar el esfuerzo que supone aprender o las consecuencias de una mala calificación, esto es, si se busca minimizar los costos, los alumnos se esforzarán lo justo para aprobar. Si por el contrario lo que les interesa es aprender, no sólo se esforzarán más, sino que lo harán utilizando estrategias orientadas a facilitar la comprensión y la adquisición de competencias. Pero aunque las metas a conseguir sean atractivas, las personas no nos esforzamos si nuestras expectativas de conseguirlas no son altas, y el que esto ocurra depende de que sepamos cómo afrontar las tareas y problemas necesarios para aprender - lo importante no son las tareas, problemas, etc., sino lo que se aprende al realizarlas y que permitirá resolver nuevos problemas en el futuro- - A veces no es que los estudiantes no aprendan porque no se esfuerzan, sino que no se esfuerzan porque cuando lo hacen no experimentan progreso, normalmente porque no saben cómo afrontar la tarea, porque no saben "pensar". Estos hechos sugieren que para motivar lo que hay que hacer es ayudar a que el alumno experimente progreso, lo que nos debe hacer revisar el planteamiento de las actividades de aprendizaje y el tipo de ayudas que proporcionamos.

\section{Características de los modos de evaluar}

Existe numerosa evidencia de que los modos de evaluación preferidos por los profesores tienen numerosos defectos (Black \& William, 1998; Crooks, 1988; Segers, Dochy \& Cascallar, 2003; Villa \& Alonso-Tapia, 1996). De hecho, muchas de las tareas utilizadas no permiten identificar el origen de las dificultades de los alumnos, razón por la que no se les puede ayudar a superar las dificultades mediante una retroalimentación precisa (Birenbaum y otros, 2006). Este hecho es particularmente preocupante cuando está demostrado que la retroalimentación frecuente y precisa es uno de los factores que más contribuye a la mejora de la motivación (Black \& William, 1998; Mayer, 2004; Kirschner, Sweller y Clark, 1996), y que cuando no se produce, los efectos son negativos, como se expone a continuación.

\section{Efecto de la evaluación en la motivación.}

Las características con que los alumnos afrontan las actividades de aprendizaje se activan dependiendo de cómo los profesores configuran los entornos de aprendizaje, esto es:

a) de lo que hacen para crear la intención de aprender,

b) de lo que hacen para mantener el interés y el esfuerzo, y

c) del modo en que plantean la evaluación, punto en el que nos vamos a centrar.

Varias revisiones recientes han tratado los efectos de la evaluación sobre la motivación, la comprensión y el cambio conceptual (Birenbaum y otros 2006; Harlen \& Crick, 2003). Estos últimos autores han revisado la evidencia proporcionada por 19 estudios para respon- 
der a la pregunta: ¿cuál es el impacto de la evaluación del aprendizaje, realizada tanto por los profesores como por las autoridades académicas mediante tests, sobre la motivación de los alumnos por aprender?, revisión que peso de manifiesto lo siguiente:

1) A menudo se produce un desajuste entre el modo de aprender preferido por los alumnos y el modo de enseñar y evaluar utilizado por los profesores, desajuste que afecta a la percepción de habilidad, al autoconcepto y a la autoestima. Muchos alumnos prefieren un aprendizaje basado en la experiencia práctica, pero los modos de evaluar y enseñar priorizan un aprendizaje muy pautado y secuenciado que parece favorecer a las alumnas.

2) La evaluación externa seguida de fracaso, especialmente cuando se utiliza para señalar a los profesores que tienen que conseguir que sus alumnos rindan más, hace que se evalúe de modo frecuente y con pruebas semejantes a las pruebas externas, y a que se trabajen menos las materias que no son objeto de evaluación externa, hecho que influye de modo negativo en la autoestima de los que tienen dificultades con los tipos de pruebas a superar

3) La evaluación frecuente en clase seguida de información sobre el resultado favorece la acumulación de experiencias repetidas de fracaso que generan una gran ansiedad ante las pruebas de evaluación, ansiedad que influye en las estrategias de afrontamiento que los alumnos adoptan, y que supone un incremento de la atención a los resultados y no al proceso.

4) En cuanto a los efectos de la cantidad y tipo de retroalimentación que los alumnos reciben tras la evaluación, los resultados han puesto de manifiesto que la información del "resultado" afecta a la percepción de capacidad — a menudo negativamente- $\mathrm{y}$, además, que interactúa con la orientación motivacional en la determinación del esfuerzo a realizar.

5) Si en lugar de recibir retroalimentación a partir de la evaluación realizada por los profesores, el sistema de clase está planteado de forma que facilita la autoevaluación y la autorregulación del aprendizaje, los alumnos tienden a centrarse más en la tarea y menos en el resultado y en la calificación del mismo, hecho que es positivo.

6) Además, se ha comprobado que la retroalimentación y la ayuda tras la evaluación influyen muy positivamente en las expectativas de autoeficacia y en el esfuerzo consiguiente.

7) Crear condiciones que favorezcan la autoevaluación como, por ejemplo, hacer que los alumnos repitan mentalmente el objetivo de aprendizaje a conseguir con la tarea durante su realización, influye en la mejora del rendimiento.

8) La evaluación centrada en la calificación contribuye a que con el aumento de la edad los alumnos estén más pendientes de los resultados que del proceso de aprendizaje, y a que los alumnos con peores logros tiendan a responder a los tests contestando aleatoriamente.

No obstante, a medida que los estudiantes se familiarizan con procedimientos como la evaluación de portafolios en el contexto de la enseñanza basada en problemas u organizada por proyectos, su actitud hacia las mismas cambia, aunque no siempre (Birenbaum y otros, 2006). La clave parece estar en el hecho de que los modos de evaluación propuestos: 
a) traen a primer plano que la meta que está en juego es aprender y no conseguir tal o cual resultado,

b) permiten organizar y distribuir la propia actividad de modo que no se percibe que aprender tiene un costo elevado, $y$,

c) posibilitan una retroalimentación regular que mejorar el progreso.

En resumen, parece un hecho claro que la evaluación, tal y como suele darse influye en la orientación hacia el resultado, en el descenso de las expectativas de autoeficacia y del esfuerzo por aprender, así como en el empeoramiento del autoconcepto y la autoestima. Cabe, pues preguntarse: ¿Qué pueden hacer los profesores, para evitar los efectos negativos de la evaluación sobre la motivación y el aprendizaje? Y también, ¿en qué dirección se debería trabajar desde los servicios de orientación?

\section{Problemas para el cambio en los modos de evaluación}

La respuesta a la pregunta anterior aparentemente parece fácil. Tal y como han sugerido Harlen y Crick (2003) y Birenbaum y otros (2006) y Dochy (2005) y como hemos mostrado en un trabajo reciente (Alonso Tapia, 2005b), por lo que a la evaluación de clase se refiere bastaría con que la evaluación se integrase en el proceso de aprendizaje en lugar de hacerse básicamente al final, con que fuese seguida regularmente de retroalimentación sobre cómo superar las dificultades y con que se estimulase la autoevaluación — no la autocalificación - y todo ello en el contexto de un proceso orientado a la adquisición de competencias percibidas relevantes en donde el alumno tuviese la posibilidad de autorregular su aprendizaje en vez de tener que apoyarse en la regulación establecida por sus profesores. Por otra parte, por lo que se refiere a las evaluaciones de tipo sumativo, sería preciso que fuesen coherentes con los modos de evaluación empleados en clase, que se utilizasen para dar retroalimentación, y que posibilitasen también el desarrollo de la capacidad de autoevaluación y uso de las competencias adquiridas. Sin embargo, el problema no es tan sencillo.

Es posible hacer evaluaciones frecuentes e integrarlas en el proceso de aprendizaje, aunque es un hecho poco habitual en los profesores de secundaria y bachillerato (Black y Wi1liam, 1998; Villa y Alonso Tapia, 1996). Sin embargo, lo que resulta más difícil a la luz de la evidencia de que disponemos es que puedan servir de base para una retroalimentación que ayude a los alumnos a superar las dificultades debido a los dos hechos siguientes:

\section{Ideas de los profesores sobre cómo es adecuado evaluar}

Muchos profesores no parecen tener conocimientos claros sobre los factores cognitivos que influyen en las dificultades que los alumnos experimentan en relación con la comprensión y las demás competencias a adquirir. Este hecho hace que las tareas empleadas para la evaluación no constituyan criterios válidos de aprendizaje ni permitan una retroalimentación capaz de ayudar a superar las dificultades, como demuestran un total de 20 estudios realizados por nuestro grupo (Alonso Tapia, Asensio, López y Carriedo, 2004).

En los estudios a que nos referimos se procedió de manera similar. Para facilitar la tarea y la comparación de los resultados se proporcionaba a los profesores una base común —un conjunto de conceptos, un texto, una tabla, un gráfico, etc.- , y se les pedía que diseñasen 
una tarea que considerasen adecuada para evaluar si los alumnos poseían o no una determinada competencia de las que, de acuerdo con el diseño curricular vigente en el momento de recoger los datos, se debía intentar que los alumnos adquiriesen trabajando en su área. Una vez recogidos los datos, a fin de poder analizarlos se desarrollaron una serie de códigos basados en los modelos que la psicología ofrecía sobre los factores que definían y determinaban cada una de las competencias analizadas, códigos que sirvieron para clasificar las tareas de evaluación propuestas por los profesores y, posteriormente, para valorar su adecuación. Ilustramos estos estudios con un ejemplo.

Una de las competencias a evaluar es la capacidad de comprender textos. Se sabe que comprender un texto implica construir una doble representación - proposicional y situacional- de su contenido, representación que depende de diversas inferencias que los alumnos tienen que hacer durante el proceso de lectura. En consecuencia, si los profesores quieren evaluar si los alumnos comprenden un texto de su área y, en caso negativo, de dónde surgen las dificultades para poder dar las ayudas precisas, deberían evaluar si los alumnos comprenden el vocabulario, qué elaboración realizan de las distintas ideas, en qué medida las conectan siguiendo la progresión temática del texto, en qué medida distinguen el distinto grado de importancia de las ideas, en qué grado elaboran una representación situacional adecuada y son capaces de identificar la intención del autor, etc. Pues bien, una vez codificadas las respuestas de los profesores, nos encontramos con los resultados que aparecen en la tabla 1.

TABLA 1: Porcentaje de profesores que sugieren cada tipo de categoría de preguntas.

\begin{tabular}{|l|c|c|c|}
\hline \multicolumn{1}{|c|}{ Categorías de preguntas } & \multicolumn{2}{c|}{ \% de profesores que las usan } \\
\cline { 2 - 4 } & $\begin{array}{c}\text { Total } \\
\text { N: } \mathbf{6 8}\end{array}$ & $\begin{array}{c}\text { España } \\
\text { N: } \mathbf{3 8}\end{array}$ & $\begin{array}{c}\text { Brasil } \\
\text { N: } \mathbf{3 0}\end{array}$ \\
\hline 1. Preguntas sobre el significado de las palabras & 72 & 89 & 57 \\
\hline 2. Preguntas sobre ideas de poca importancia & 21 & 25 & 17 \\
\hline $\begin{array}{l}\text { 3. Preguntas sobre proposiciones que transmiten información } \\
\text { importante. }\end{array}$ & 19 & 23 & 14 \\
\hline $\begin{array}{l}\text { 4. Preguntas sobre la idea principal. } \\
\text { 5. Preguntas sobre el modelo de situación al que se refiere el } \\
\text { texto }\end{array}$ & 29 & 32 & 27 \\
\hline 6. Preguntas sobre la intención del autor. & 29 & 50 & 10 \\
\hline 7. Preguntas sobre la valoración crítica del contenido del texto & 5 & 0 & 10 \\
\hline $\begin{array}{l}\text { 8. Preguntas de recuerdo de información no necesaria para } \\
\text { entender el texto }\end{array}$ & 47 & 54 & 40 \\
\hline
\end{tabular}

Como puede comprobarse, sólo un $12 \%$ de los profesores considera que plantear preguntas sobre la idea principal es necesario si se quiere evaluar la comprensión del texto, porcentaje que se eleva al $29 \%$ en el caso del modelo de situación, señalando la mayoría 
— un $72 \%$ - la necesidad de preguntar por el significado de las palabras. Sin embargo, un $47 \%$ sugiere el planteamiento de preguntas que podrían haberse respondido incluso sin leer el texto, recordando sólo la información sobre el tema. Obviamente, con este tipo de preguntas es difícil que un profesor pueda plantear sugerencias y proporcionar ayuda adecuada a los alumnos que experimentan dificultades de comprensión, con lo que la repercusión motivacional de la evaluación seguiría las pautas descritas en el apartado anterior. Resultados análogos a los descritos se han encontrado en los veinte estudios realizados.

\section{Reacciones a los procesos de formación}

Llegados a este punto se podría pensar que la solución del problema pasa por una formación adecuada del profesorado. Sin embargo, aquí interviene el segundo hecho al que habíamos aludido. Sobre la base de los resultados anteriores, Alonso Tapia, Asensio López y Carriedo (2004) desarrollaron y valoraron un programa de formación en evaluación dirigido a profesores de Ciencias Sociales, valoración cuyos resultados pusieron de manifiesto varios hechos importantes.

En primer lugar y en línea con lo esperado, el cambio experimentado por los profesores fue estadística y psicológicamente significativo - el porcentaje medio de ganancia fue del $32 \%$ - Así pues, las competencias necesarias para una evaluación adecuada que facilite la retroalimentación precisa sobre el origen de las dificultades y, a través de ella, la motivación, se pueden adquirir con una formación adecuada y sin demasiado problema.

En segundo lugar, el programa fue valorado muy positivamente en su conjunto y en cada módulo por los profesores en todos sus aspectos - coherencia con los objetivos de la materia, claridad, utilidad, adecuación de la metodología, y posibilidad de mejorar el aprendizaje y la motivación de los alumnos- Sin embargo, y esto es lo importante para el tema que nos interesa, la aplicabilidad del programa recibió sólo una puntuación promedio correspondiente a "suficiente". Las dificultades a superar para conseguir una mayor aplicabilidad radican sobre todo, de acuerdo con las sugerencias de los profesores, en que lleva mucho tiempo preparar tareas como las que se proponen, por lo que constituiría una ayuda altamente facilitadora el que pudiesen disponer de un banco de tareas de las que poder echar mano, al menos en principio.

Sin embargo, en el marco de los resultados comentados cabe preguntarse cómo afectaría a la motivación el que los profesores llegasen a evaluar siguiendo las pautas indicadas. Averiguarlo es el objeto de este estudio de carácter piloto, dado que se centra en una única asignatura y es de tipo básicamente descriptivo, orientado a permitir posteriormente el diseño de estudios de mayor profundidad y rigor metodológico.

\section{MÉTODO}

Muestra. La muestra del estudio la constituyeron 113 alumnos, 45 chicas y 68 chicos, de $3^{\circ}$ de Educación Secundaria, con una media de 14,65 años de edad. Pertenecían a un colegio concertado de Madrid. El mismo profesor enseñaba Geografía a todos ellos. 


\section{Procedimiento}

Frente al trabajo que los alumnos realizaban habitualmente, centrado en la asimilación de los contenidos explicados por el profesor y evaluados mediante pruebas objetivas con cuatro alternativas que ponían en juego sobre todo - aunque no exclusivamente- el recuerdo de los conocimientos adquiridos, durante el período del estudio trabajaron los temas correspondientes a la penúltima evaluación del curso mediante tareas que requerían resolver problemas que implicaban analizar datos, categorizarlos desde los conceptos y modelos vistos en clase y hacer inferencias de tipo predictivo, como ilustra el ejemplo que se incluye en la tabla 2. Posteriormente se realizó la prueba de evaluación con cuatro tareas análogas a las descritas en la tabla mencionada. Una vez realizada ésta, se pasó a los alumnos un cuestionario cuyas preguntas que debían ser contestadas en una escala de 1 a 7 puntos, cada uno

TABLA 2: Ejemplo de prueba para evaluar comprensión.

Los siguientes países presentan unas características determinadas en relación con las actividades del sector primario. Responde de forma razonada a las siguientes preguntas fijándote en la tabla.

\begin{tabular}{|l|c|c|c|c|}
\hline \multicolumn{1}{|c|}{ Factores } & PAIS 1 & PAIS 2 & PAIS 3 & PAIS 4 \\
\hline Precipitaciones abundantes & NO & SI & SI & NO \\
\hline Relieve abrupto & SI & NO & NO & SI \\
\hline Escaso desarrollo económico & $\mathrm{SI}$ & $\mathrm{SI}$ & NO & NO \\
\hline Alta densidad de población & NO & SI & SI & NO \\
\hline Suelos buenos & NO & SI & SI & NO \\
\hline Buenas infraestructuras de transporte & NO & NO & SI & SI \\
\hline
\end{tabular}

1. ¿Crees que en alguno de estos países la población ha cultivado en bancales de forma generalizada a lo largo de su historia? ¿En cuál de ellos? ¿Por qué?

2. ¿En cuál de estos países crees más probable que los agricultores cultiven en invernaderos de forma intensiva? ¿Por qué?

3. Hay dos países en los que predominaría el policultivo y las parcelas pequeñas. ¿Cuáles son? ¿Qué dos factores explican este hecho de los seis que aparecen en la tabla?

4. El barbecho y el regadío son dos técnicas agrícolas bastantes extendidas. Di dos países en los que crees que se practicaría de forma generalizada cada una de ellas razonando tu respuesta.

5. ¿Hay algún país en el que se practicaría de forma generalizada el tipo de agricultura de subsistencia denominado "itinerante por cremación", "de rocas" o de "corta y quema"? Razona tu respuesta.

6. Responde a la pregunta anterior pero en el caso de los siguientes tipos de agricultura de subsistencia: "agricultura extensiva de secano" y "agricultura irrigada del arroz". Explica tu respuesta en ambos casos, citando al menos dos factores que te llevan a dar tu respuesta.

7. ¿En qué dos países crees más probable la aparición y desarrollo de ganadería de subsistencia? ¿Qué tipos de ganadería de los tres vistos durante las explicaciones se llevaría a cabo en cada uno de estos países?

8. ¿En cuál de estos países se llevaría a cabo un tipo de ganadería estabulada e intensiva? Cita dos factores de los seis que caracterizan a ese país y que te llevan a dar tu respuesta. 
de los cuales estaba definido por un adjetivo. En todas las preguntas excepto en la B4 y la $\mathrm{C} 4$, la escala era bipolar — un 4 indicaba efecto nulo-. En estas dos últimas preguntas, por el contrario, la preferencia aumenta con la puntuación. Las preguntas del cuestionario aparecen en la tabla 3. El objetivo de las preguntas del cuestionario era por una parte, examinar, la percepción del impacto motivacional que suponía para los alumnos el tener que trabajar antes de dar un tema, durante el mismo y al hacer la evaluación con tareas como las que hemos presentado en la tabla 2, impacto motivacional evaluado en términos de interés, esfuerzo por aprender y preferencia por las nuevas tareas; y, por otra parte, obtener información sobre las expectativas de autoeficacia específica a la hora de enfrentarse a tales tareas y de conseguir una calificación positiva, dado que la novedad de las tareas podía influir en estas variables y afectar a la valoración que los alumnos hiciesen del nuevo método de evaluación.

TABLA 3: Valor motivacional de las tareas utilizadas. Medias y desviaciones típicas.

\begin{tabular}{|c|c|c|c|}
\hline Preguntas & Media & Sd & Significado \\
\hline $\begin{array}{l}\text { A. Uso de las tareas antes de la enseñanza. } \\
\text { ¿Disminuiría o aumentaría tu interés por la tarea? } \\
\text { ¿Te motivaría a esforzarte por aprender y no sólo por } \\
\text { aprobar? }\end{array}$ & 4,64 & 1,05 & $\begin{array}{l}\text { Aumentaría algo } \\
\text { Aumentaría algo }\end{array}$ \\
\hline $\begin{array}{l}\text { B. Uso de las tareas durante el proceso de aprendizaje. } \\
\text { ¿Crees que te sería fácil resolver este tipo de tareas? } \\
\text { ¿Disminuiría o aumentaría tu interés por la tarea? } \\
\text { ¿Te motivaría a esforzarte por aprender y no sólo por } \\
\text { aprobar? } \\
\text { ¿Preferirías que te planteasen tareas como éstas? }\end{array}$ & $\begin{array}{l}4,29 \\
5,08 \\
5,16 \\
3,92\end{array}$ & $\begin{array}{l}1,39 \\
1,09 \\
0,97 \\
1,62\end{array}$ & $\begin{array}{l}\text { Ni F ni D } \\
\text { Aumentaría algo } \\
\text { Aumentaría algo } \\
\text { Bastantes veces }\end{array}$ \\
\hline $\begin{array}{l}\text { C. Uso de las tareas para la evaluación. } \\
\text { ¿Crees que te sería fácil sacar una nota positiva? } \\
\text { ¿Disminuiría o aumentaría tu interés por la tarea? } \\
\text { ¿Te motivaría a esforzarte por aprender y no sólo por } \\
\text { aprobar? } \\
\text { ¿Preferirías que te planteasen tareas como éstas? }\end{array}$ & $\begin{array}{l}4,16 \\
4,67\end{array}$ & $\begin{array}{l}1,42 \\
1,25\end{array}$ & $\begin{array}{l}\text { Ni F ni D } \\
\text { Aumentaría algo } \\
\text { Aumentaría algo } \\
\text { Bastantes veces }\end{array}$ \\
\hline
\end{tabular}

\section{Hipótesis y análisis realizados}

En primer lugar, se comprobó si había habido cambios en el rendimiento — calificaciones - entre dos evaluaciones sucesivas, para lo que tras calcular las medias y desviaciones típicas se comprobó si las diferencias eran significativas. La expectativa en este caso, al tratarse de efectos a corto plazo y de que las tareas eran novedosas, era que las puntuaciones fuesen algo menores tras el entrenamiento y pese al mismo. En segundo lugar se realizó un análisis descriptivo de los resultados correspondientes al valor motivacional atribuido por loas alumnos al uso de las nuevas tareas tanto para el aprendizaje como para la evaluación. En este caso esperábamos que los alumnos valorasen positivamente las tareas, aunque 
dada su novedad y la posibilidad de fracaso por la falta de práctica con las mismas, la seguridad que teníamos de lo acertado de nuestras expectativas no era muy grande. Finalmente, analizamos las correlaciones entre el valor motivador que los alumnos habían atribuido al uso de las nuevas tareas en los distintos momentos del proceso de aprendizaje y la preferencia por las mismas, y las expectativas de autoeficacia y de resultado generadas específicamente por tales tareas.

\section{Resultados}

En primer lugar, el análisis de varianza de medidas repetidas de las medias entre las dos evaluaciones sucesivas (Antes: $\mathrm{Md}=6.25, \mathrm{Sd}=1.42$; Después: $\mathrm{Md}=5.50, \mathrm{Sd}=1,66$ ) puso de manifiesto que, pese a que la correlación entre ambos conjuntos de puntuaciones fue significativa $(\mathrm{r}=403, \mathrm{p}<0.001)$, el rendimiento había disminuido significativamente $\left(\mathrm{F}_{\mathrm{gl}=112}=22.09 ; \mathrm{p}<0.0001\right)$. Como esperábamos, tras una primera experiencia con procedimientos de trabajo y evaluación con los que no se está habituado el rendimiento fue menor.

Ante estos resultados, cabría esperar que los alumnos evaluasen negativamente las tareas utilizadas, evaluación cuyos resultados recoge la tabla 3, donde se presentan las medias y desviaciones típicas de las puntuaciones correspondientes a las respuestas. Los datos, sin embargo, ponen de manifiesto que tanto el interés como la motivación para esforzarse por aprender aumentarían algo si se empleasen el tipo de tareas sugeridas, y que preferirían que los profesores las utilizasen bastante a menudo. Estos resultados son alentadores aunque no demasiado si bien hay datos que explican porqué las puntuaciones no son más altas.

El análisis de las correlaciones entre la percepción de autoeficacia para resolver los problemas y las expectativas de conseguir resultados positivos, por un lado, y las puntuaciones en el interés, la motivación y la preferencia por el tipo de tareas utilizadas por otro ha puesto de manifiesto una fuerte asociación entre las puntuaciones en estos dos grupos de variables, como muestra la tabla 4.

TABLA 4: Correlaciones entre interés, motivación y preferencia por las tareas con las expectativas de autoeficacia y de resultado

\begin{tabular}{|l|c|c|c|c|c|c|c|c|}
\hline \multirow{2}{*}{ Expectativas } & \multicolumn{5}{c|}{ Motivación } \\
\cline { 2 - 9 } & \multicolumn{2}{|c|}{ Antes } & \multicolumn{3}{c|}{ Durante } & \multicolumn{3}{c|}{ Evaluación } \\
\hline $\mathbf{R}_{\text {eficacia-resultado }}, \mathbf{6 4 2 ^ { * * }}$ & Interés & Motivación & Interés & Motivación & Preferencia & Interés & Motivación & Preferencia \\
\hline Eficacia &, 147 &, $218^{*}$ &, $307^{* *}$ &, $236^{*}$ &, $651^{* *}$ &, $381^{* *}$ &, $343^{* *}$ &, $531^{* *}$ \\
\hline Resultado &, 108 &, 103 &, 146 &, 176 &, $520^{* *}$ &, $399^{* *}$ &, $399^{* *}$ &, $625^{* *}$ \\
\hline
\end{tabular}

* Significativo al .05\%; $\quad$ ** Significativo al .01\%

El conjunto de los resultados presentados sugiere - aunque no demuestra, por ser éstos de tipo correlacional - que pese a que la introducción de tareas más relevantes y que permiten una retroalimentación más precisa para ayudar a los alumnos a mejorar tiene un efecto positivo en la motivación, este efecto es muy limitado. Es positivo porque, pese a que las notas han sido peores con el nuevo procedimiento de evaluación, los alumnos prefieren los 
nuevos procedimientos. Y limitado, porque sólo en la medida en los alumnos lleguen a experimentar que pueden progresar — sólo si mejoran sus expectativas de autoeficacia- y que sus calificaciones no van a sufrir - si mejoran sus expectativas de resultado- su valoración de estas pruebas tiende a mejorar. Este es un resultado en cierto modo esperable, dado

a) que se trataba de una experiencia de trabajo y de evaluación novedosa,

b) que el cuestionario se les pasó antes de recibir retroalimentación de la evaluación y

c) que los alumnos seguían trabajando en el conjunto de las materias en un contexto marcadamente orientado a los resultados.

\section{Discusión y conclusiones}

Creemos que el conjunto de hechos presentados deja claro que uno de los pasos que hay que dar para mejorar la motivación de los alumnos es modificar las formas actuales de evaluación, de modo que los profesores evalúen "para" el aprendizaje en lugar de evaluar "el aprendizaje". Hemos puesto de manifiesto, sin embargo, que no es una tarea sencilla debido a tres hechos.

1) A que hay que comenzar no tanto por enseñar estrategias como la evaluación de portafolios u otros procedimientos más ligados al proceso de aprendizaje y que permitan una retroalimentación más frecuente, cuanto por ayudar al profesorado a reflexionar sobre la adecuación de las tareas que constituyen la base misma de la evaluación y los criterios para valorarlas.

2) Incluso sabiendo cómo hacer una evaluación adecuada y alternativa a la actual, el coste que supone el diseño de tareas adecuadas exige un apoyo en forma de materiales tanto para la evaluación como para la instrucción previa en consonancia con la misma que facilite trabajo del profesorado, aunque nunca lo sustituya plenamente.

3) Incluso si los profesores adoptan nuevas formas de trabajo y evaluación en la línea indicada, si no cambia el resto del clima de clase, de manera que la retroalimentación y apoyo frecuente haga que los alumnos experimenten progreso y aumenten sus expectativas de autoeficacia y de buenos resultados, el impacto motivacional será limitado.

Estos hechos plantean un triple desafío, a saber:

1) Fundamentar y desarrollar modelos de evaluación que mejoren la motivación y el aprendizaje;

2) fundamentar y desarrollar modelos de formación del profesorado — en preparación o en ejercicio - para que adquieran las competencias en evaluación señaladas; y

3) desarrollar instrumentos y material que faciliten la puesta en práctica de los nuevos modelos de evaluación.

Sin responder a los desafíos anteriores es difícil —especialmente a partir de la enseñanza secundaria, dados los hábitos de evaluación de los profesores- que éstos cambien de modo que la evaluación influya positivamente en la motivación. A estos desafíos hay que añadir que se requerirá tiempo y apoyo a los alumnos para que la utilización de las nuevas formas de evaluación cambien las expectativas de éstos de modo que lleguen a valorarlas positivamente. 
¿Qué pueden hacer los orientadores a la luz de las ideas expuestas? Como hemos señalado en otro artículo (Alonso Tapia, 2001), quizás lo primero deba ser ayudar a los profesores a tomar conciencia de que los problemas de los alumnos no están sólo en éstos, sino en el tipo de entorno de aprendizaje que creamos con nuestras formas de organizar y desarrollar la enseñanza, entorno en el que las tareas de evaluación tienen un peso especial pues definen el tipo de uso que los alumnos han de hacer del conocimiento y, en consecuencia, qué es lo que tienen que esforzarse por aprender, objetivos que a menudo resultan poco significativos para los propios alumnos. Para ello, cuando los profesores o las profesoras les planteen problemas de desmotivación, conviene hacerles pensar sobre las implicaciones que los modos de evaluar que estén utilizando pueden estar teniendo sobre la motivación y el aprendizaje de sus alumnos.

Además, en espera de las nuevas aportaciones que pueda hacer la investigación, es preciso plantear a los profesores - individualmente o en grupo - la necesidad de evaluar "para el aprendizaje" y de buscar mediante la reflexión y la experimentación medios de evaluación que permitan una retroalimentación más continua, cualitativamente más precisa, sobre competencias cuya adquisición resulte significativa a los alumnos $\mathrm{y}$, en la medida de lo posible, que les permita interiorizar criterios desde los que autoevaluarse, para lo que puede ser de utilidad consultar y reflexionar sobre algunas publicaciones que abordan este problema (Alonso Tapia, 1997; 2005c; Segers, Dochy y Cascallar, 2003; Birenbaum y otros, 2006). Como indicaba el título de este artículo y como han mostrado los datos sobre el modo en que los cambios en la evaluación pueden mejorar la motivación, es preciso que los profesores, al reflexionar, lo hagan pensando en que los medios de evaluación utilizados deben contribuir a que los alumnos comprendan y valoren la utilidad de adquirir los conocimientos y competencias que se están evaluando pero, sobre todo, que experimenten que aprenden y progresan y que pueden seguir haciéndolo, de modo que vean que el esfuerzo merece la pena.

\section{Referencias bibliográficas}

Alonso-Tapia, J. (Dir.) (1997). Evaluación del conocimiento y su adquisición. (Vol. 1: Ciencias Sociales; Vol. 2 Ciencias Naturales y Experimentales; Vol 3. Matemáticas y Comprensión Lectora). Madrid: Ministerio de Educación y Ciencia.

Alonso Tapia, J. (2001). "La evaluación de la competencia curricular en el contexto de la orientación educativa”. Revista de Española de Orientación y Psicopedagogía, 12 (21), 15-38.

Alonso Tapia, J. (2005a). "Motivaciones, expectativas y valores-intereses relacionados con el aprendizaje: Nuevas perspectivas sobre un antiguo problema a partir del desarrollo y validación del cuestionario". Psicothema, Vol. 17, 3, 404-411.

Alonso Tapia, J. (2005b). Motivar en la escuela, motivar en la familia. Madrid, Morata.

Alonso Tapia, J., Asensio, F., López, I. y Carriedo (2004). "Evaluación del conocimiento y formación del profesorado". En Ministerio de Educación, Cultura y Deporte (Ed.), Premios Nacionales de Investigación Educativa 2002. (pp. 157-204). Madrid, MEC.

Alonso Tapia, J. y Ruiz, M. A. (2007). "Motivation, self-regulation and academic achievement: A study on their relationships and educational implications coming from the structural and predictive validity of the MEVA questionnaire". Psicothema, 19, 602-608. 
Birenbaum, M., Breuer, K. Cascallar, E., Dochy, F., Ridgway, J., Wiesemes, R. \& West, A. (2006). "A learning integrated assessment system". Educational Research Review, 1, 1, 61-67.

Black, P. \& William, D. (1998). “Assessment and classroom learning”. Assessment in Education: Principles, Policy \& Practice, 5, 7-74.

Crooks, T. J. (1988). "The impact of classroom evaluation practice on students". Review of Educational Research, 58, 348-381.

Dochy, F. (2005). "Learning lasting for life" and assessment: How far did we progress? Conferencia Presidencial en el 20 aniversario de la European Association for Research on Learning and Instruction. Nicosia.

Eccles, J. S. y Wigfield, A. (2002). "Motivational beliefs, values and goals". Annual Review of Psychology, 53, 109-132.

Harlen, W. y Crick, R. D. (2003). "Testing and motivation for Learning”. Assessment in education, 10, 2, 169-207.

Kirschner, P. A., Sweller, J. y Clark, R. E. (2006). "Why minimal guidance during instruction does not work: An analysis of the failure of constructivist, discovery, problem-based, experiential and inquiry-based teaching”. Educational Psychologist, 41(2), 75-86.

Mayer, R. (2004). "Should there be a three-strikes rule against pure discovery learning? The case for guided methods of instruction". American Psychologist, 59, 1, 14-19.

Segers, M., Dochy, F. \& Cascallar, E. (2003). Optimizing new modes of assessment: In search for qualities and standards. Boston: Kluwer.

Villa, J. L. \& Alonso Tapia, J. (1996). "Evaluación del conocimiento: Procedimientos utilizados por los profesores en BUP y FP”. En Ministerio de Educación y Ciencia (Ed.), Premios Nacionales de Investigación e Innovación Educativa 1994. (pp. 51-78). Madrid: Ministerio de Educación y Ciencia, CIDE.

Fecha de recepción: 24-05-06

Fecha de revisión: 06-06-07

Fecha de aceptación: 07-11-07 